\title{
Structures of Synthetic Nanobodies in Complex with SARS-CoV-2 Spike or Receptor-Binding Domain Provide Insights for Developing Therapeutics and Vaccines
}

\author{
J Jiang ${ }^{1}$, J A Dhobi ${ }^{2}$, K Natarajan ${ }^{3}$, L F Boyd ${ }^{4}$, R Huang ${ }^{5}$, A Zeher ${ }^{6}$, D H Margulies ${ }^{7}$ \\ ${ }^{1} \mathrm{NIAID/NIH}{ }^{2} \mathrm{LISB} / \mathrm{NIAID/NIH},{ }^{3} \mathrm{LISB} / \mathrm{NIAID/NIH},{ }^{4} \mathrm{LISB} / \mathrm{NIAID/NIH},{ }^{5}$ Laboratory of Cell \\ Biology/CCR/NCI/NIH, ${ }^{6}$ Laboratory of Cell Biology/CCR/NCI/NIH, ${ }^{7}$ LISB/NIAID/NIH \\ jiangji@niaid.nih.gov
}

\begin{abstract}
Rapid structure determination of antibodies or nanobodies complexed with SARS-CoV-2 spike or its receptorbinding domain (RBD) by X-ray crystallography and cryo-EM provide structural insight for developing effective therapies and vaccines to combat the current pandemic and recent circulating variants of the virus. We report crystal structures of synthetic nanobodies (sybody) Sb16, Sb45, and Sb68 bound to the RBD of SARS-CoV-2: two binary complexes of Sb16/RBD and Sb45/RBD; a ternary complex of Sb45+RBD+Sb68 [1,2]; and a cryo-EM structure of $\mathrm{Sb} 45$ bound to the trimeric spike protein. Sb16 and Sb45 bind the RBD at the angiotension converting enzyme (ACE)2 interface, positioning their complementarity determining region (CDR) CDR2 and CDR3 loops in opposite orientations diametrically, and recognizing distinct epitopic areas. The buried surface area (BSA) at sybody/RBD interfaces is consistent with neutralization activity. A large shift of the CDR2 loop is identified by comparing Sb16 alone (unliganded) with Sb16/RBD (complex) structures. Structural analysis based on these and more than 100 recent structures of antibodies, nanobodies, and sybodies in complex with RBD or spike reveals the vital role of CDR loops and their dynamic flexibility in recognizing epitopes. Superposition of these crystal structures onto cryoEM structures of trimeric spike protein indicates that the three sybodies can access both "up" and "down" conformations of the mature spike. Cryo-EM maps of Sb45/spike complexes confirm these models. SARS-CoV-2 variants B.1.1.7 (UK) and B.1.351 (South Africa), which have increased affinity for the cellular receptor ACE2, appear to be less susceptible to human antibodies elicited by current mRNA vaccines (Moderna and PfizerBioNTech). Our binding studies indicate that the common RBD escape mutant N501Y is still bound by Sb16 and $\mathrm{Sb} 45$, although with lower affinity. The E484K mutation, however, has a much lower affinity for Sb16 and Sb45 due to the loss of hydrogen-bonding networks. Insights provided by structural analysis of these complexes of sybodies with RBD or spike may contribute to developing new therapeutics and to updating the vaccine.
\end{abstract}

(Supported by the Intramural research program of the NIAID/NIH and NIH IRP Cryo-EM Facility)

References:

[1] Ahmad, Jiang \& Margulies et al. 2021, bioRxiv, https://www.biorxiv.org/content/10.1101/2021.01.27.428466v1

[2] Walter \& Seeger et al. 2020, bioRxiv, https://www.biorxiv.org/content/10.1101/2020.11.10.376822v1, 